\title{
Distributed Exact Weighted All-Pairs Shortest Paths in Near-Linear Time
}

\author{
Aaron Bernstein ${ }^{1}$ and Danupon Nanongkai ${ }^{2}$ \\ ${ }^{1}$ Rutgers University. bernstei@gmail.com \\ ${ }^{2}$ KTH Royal Institute of Technology, Sweden. danupon@gmail.com
}

\begin{abstract}
In the distributed all-pairs shortest paths problem (APSP), every node in the weighted undirected distributed network (the CONGEST model) needs to know the distance from every other node using least number of communication rounds (typically called time complexity). The problem admits $(1+o(1))$-approximation $\tilde{\Theta}(n)$-time algorithm and a nearly-tight $\tilde{\Omega}(n)$ lower bound [Nanongkai, STOC'14; Lenzen and Patt-Shamir PODC'15] ${ }^{1}$. For the exact case, Elkin [STOC'17] presented an $O\left(n^{5 / 3} \log ^{2 / 3} n\right)$ time bound, which was later improved to $\tilde{O}\left(n^{5 / 4}\right)$ [Huang, Nanongkai, Saranurak FOCS'17]. It was shown that any super-linear lower bound (in $n$ ) requires a new technique [CensorHillel, Khoury, Paz, DISC'17], but otherwise it remained widely open whether there exists a $\tilde{O}(n)$-time algorithm for the exact case, which would match the best possible approximation algorithm.

This paper resolves this question positively: we present a randomized (Las Vegas) $\tilde{O}(n)$-time algorithm, matching the lower bound up to polylogarithmic factors. Like the previous $\tilde{O}\left(n^{5 / 4}\right)$ bound, our result works for directed graphs with zero (and even negative) edge weights. In addition to the improved running time, our algorithm works in a more general setting than that required by the previous $\tilde{O}\left(n^{5 / 4}\right)$ bound; in our setting (i) the communication is only along edge directions (as opposed to bidirectional), and (ii) edge weights are arbitrary (as opposed to integers in $\{1,2, \ldots, \operatorname{poly}(n)\}$ ). The previously best algorithm for this more difficult setting required $\tilde{O}\left(n^{3 / 2}\right)$ time [Agarwal and Ramachandran, ArXiv'18] (this can be improved to $\tilde{O}\left(n^{4 / 3}\right)$ if one allows bidirectional communication).

Our algorithm is extremely simple and relies on a new technique called Random Filtered Broadcast. Given any sets of nodes $A, B \subseteq V$ and assuming that every $b \in B$ knows all distances from nodes in $A$, and every node $v \in V$ knows all distances from nodes in $B$, we want every $v \in V$ to know DistThrough $(a, v)=\min _{b \in B} \operatorname{dist}(a, b)+\operatorname{dist}(b, v)$ for every $a \in A$. Previous works typically solve this problem by broadcasting all knowledge of every $b \in B$, causing super-linear edge congestion and time. We show a randomized algorithm that can reduce edge congestions and thus solve this problem in $\tilde{O}(n)$ expected time.
\end{abstract}

\footnotetext{
${ }^{1} \tilde{\Theta}, \tilde{O}$ and $\tilde{\Omega}$ hide polylogarithmic factors. Note that the lower bounds also hold even in the unweighted case and in the weighted case with polynomial approximation ratios [LP13, HW12, PRT12, Nan14].
} 


\section{Contents}

1 Introduction 1

2 High-Level Overview 3

3 Preliminaries 4

3.1 The CONGEST Model . . . . . . . . . . . . . . . . . . . . . . . . . . . 4

3.2 Notation and Problem Definition . . . . . . . . . . . . . . . . 5

3.3 Distributed Algorithmic Primitives . . . . . . . . . . . . . . . 6

4 The Algorithm 6

4.1 Correctness of the Main Algorithm (Algorithm 1) . . . . . . . . . . . 7

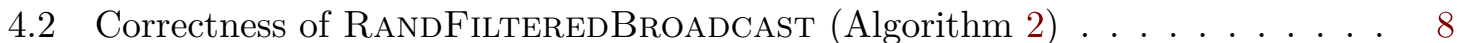

4.3 Complexity of RandFilteredBroadcast (Algorithm 2) . . . . . . . . 10

4.4 Complexity of the Main Algorithm (Algorithm 1) . . . . . . . . . . . . . 10

5 Open Problems 11

6 Acknowledgement 12

$\begin{array}{ll}\text { References } & 12\end{array}$

A Bellman-Ford 15

B Non-negative weights 16 


\section{Introduction}

We study the distributed all-pairs shortest paths problem (APSP) defined on the CONGEST model of distributed network. A network is modeled by a weighted undirected $n$-node graph $G=(V, E) .^{2}$ Each node represents a processor with unique ID and infinite computational power that initially only knows its adjacent edges and their weights. Nodes can communicate with each other in rounds, where in each round each node can send a message of size $O(\log n)$ to each neighbor (weights play no role in the communication). The goal of APSP is for every node to know its distances from all other nodes. We want an algorithm that achieves this with smallest number of rounds, called time complexity. It is usually expressed in terms of $n$ and $D$, where $n$ is the numer of nodes and $D$ is the diameter of the network when edge weights are omitted. Throughout we use $\tilde{\Theta}, \tilde{O}$ and $\tilde{\Omega}$ to hide polylogarithmic factors in $n$. See Section 3 for details of the model.

The approximate version of the problem was known to admit (i) a $(1+o(1))$-approximation $\tilde{O}(n)$-time deterministic algorithm and (ii) an $\tilde{\Omega}(n)$ lower bound which holds even against randomized $\tilde{O}(\operatorname{poly}(n))$-approximation algorithms and when $D=O(1)$ [LPS13, LP15, Nan14]. The exact unweighted version was also settled with $\tilde{\Theta}(n)$ bound [LP13, HW12, FHW12, PRT12, ACK16]. ${ }^{3}$ For the exact weighted case, nothing was known until the 2017 bound of $O\left(n^{5 / 3} \log ^{2 / 3} n\right)$ by Elkin [Elk17b], which was later improved to $\tilde{O}\left(n^{5 / 4}\right)$ [HNS17]; both algorithms by [Elk17b, HNS17] are randomized. On the lower bound side, Censor-Hillel, Khoury, and Paz [CKP17] pushed the bound to $\Omega(n)$ and proved that the standard lower bound technique cannot provide a super-linear lower bound. Despite this, it was still widely open whether there was a new technique that implies a super-linear lower bound, or whether we can in fact solve the exact weighted case in $\tilde{O}(n)$ time, like the approximate and the unweighted cases.

Our result. We present an randomized (Las Vegas) $\tilde{O}(n)$-time algorithm. This essentially settles the distributed APSP problem, with the key open remaining problem being whether deterministic algorithms can achieve the same bound. Like the previous $\tilde{O}\left(n^{5 / 4}\right)$-time algorithm, our algorithm works in a more difficult model where each node must send the same message to every neighbor in each round (broadcast CONGEST), and can handle a more general case of inputs: directed graphs with zero edge weights; in fact a standard reduction shows that our algorithm can also handle negative weights in $\tilde{O}(n)$ time.

In addition to the improved running time, our algorithm works in a more general setting than that required by the previous $\tilde{O}\left(n^{5 / 4}\right)$ bound. The previous $\tilde{O}\left(n^{5 / 4}\right)$-time algorithm of [HNS17] requires that (i) the communication is bidirectional (unaffected by edge directions), and (ii) edge weights are in $\{1, \ldots$, poly $(n)\}$. Although these are typical assumptions, some works have explored the possibilities to avoid them, e.g. [Elk17b, ARK+18, AR19, AR18] (the second assumption was also mentioned in [HNS17] as their main drawback, since their guarantee depends on the number of bits needed to represent edge weights). If we do without both assumptions (so communication is only along edge directions, and edge weights are arbitrary as long as a distance can be sent through a link in one round), the previously best

\footnotetext{
${ }^{2}$ As we will discuss later, we can also handle directed graphs.

${ }^{3}$ More precisely, the bound for the unweighted case is $\Theta(n / \log n)$. The lower bound holds against (polylog$(n)$ )-approximation algorithms when the network is unweighted. The same lower bound also holds even for the easier problem of approximating the network diameter [FHW12].
} 
algorithm for this more difficult setting required $\tilde{O}\left(n^{3 / 2}\right)$ time [AR19] ${ }^{4}$. If bidirectional communications are allowed, then the bound can be improved to $\tilde{O}\left(n^{4 / 3}\right)$ [AR18]. Our algorithm does not require any of the above assumptions, and our $\tilde{O}(n)$ bound subsumes all above results, except that the $\tilde{O}\left(n^{3 / 2}\right)$-time algorithm in [AR19] is deterministic.

Our algorithm is also much simpler than the previous $\tilde{O}\left(n^{5 / 4}\right)$ state-of-the-art. Given that our result is essentially optimal, we believe that its simplicity is a plus.

Other related works. As noted earlier, one aspect left to understand distributed APSP is the performance of deterministic algorithms. The current best time for deterministic algorithms is $\tilde{O}\left(n^{3 / 2}\right)$, first achieved by Agarwal et al. [ARK+18] and later tailored to work without bidirectional communication by Agarwal and Ramachandran [AR19]. For a summary of previous algorithms and their properties, see [AR18, Table 1].

Distributed APSP is sometimes referred to as name-independent routing schemes. See, e.g. [LPS13, LP15] for discussions and results on another variant called name-dependent routing schemes which is not considered in this paper. These papers also show an application of distributed APSP to routing tables constructions.

The previous lack of understanding for exact APSP in fact reflects a bigger issue in the field of distributed graph algorithms: Studies in the past few years have led to tight approximation algorithms for several graph problems; for example, single-source shortest paths (SSSP), minimum cut, and maximum flow can be $(1+o(1))$-approximated in $\tilde{O}(\sqrt{n}+D)$ time $[\mathrm{HKN16}, \mathrm{BKK}+17, \mathrm{Nan14}, \mathrm{NS14}, \mathrm{GK} 13, \mathrm{GKK}+15]^{5}$, and the time bounds are tight up to polylogarithmic factors [DHK+12, Elk06, PR00, KKP13, EKN+14]. In contrast, except for minimum spanning tree (e.g. [KP98, PRS17, Elk17a]), not much was known for exact algorithms until 2017, when algorithms for exact SSSP and APSP started to appear (e.g. [GL18, FN18, Elk17b, HNS17, ARK+18, AR19, AR18]). Settling the exact cases for other problems remains a major open problem.

Techniques. The cornerstone of our algorithm is a new technique called random filtered broadcasting. We give an overview in section 2 ; loosely speaking, the technique applies to settings where one needs to broadcast a large amount of information to every vertex, but in the end each vertex only cares about the "best" message it receives. We show how to use randomization to filter out most of the messages, and reduce the congestion on each edge. Although relatively simple, our result in this paper show the technique to be very powerful. It is also quite general, so we have strong reason to believe that it will find application in other distributed algorithms for the CONGEST model, especially those related to distances.

On a more concrete level, we use random filtered broadcasting to devise a primitive which leads to our APSP algorithm, but which we think may prove useful in its own right. In particular, given any sets of nodes $A, B \subseteq V$ (nodes know if they are in these sets) and assuming that every $b \in B$ knows all distances from nodes in $A$, and every node $v \in V$ knows all distances from nodes in $B$, we want every $v \in V$ to know $\operatorname{DistThrough}_{B}(a, v)=$ $\min _{b \in B} \operatorname{dist}(a, b)+\operatorname{dist}(b, v)$ for every $a \in A .{ }^{6}$ This was previously an obstacle for APSP. In this paper, we show how to do this in $\tilde{O}(n)$ time. Armed with this black-box, we are able

\footnotetext{
${ }^{4}$ We emphasize that in the case of uni-directional communication, node $v$ can learn its distance from $u$ only if there is a directed path from $u$ to $v$; otherwise, it is impossible for $v$ to learn such information.

${ }^{5}$ For the maximum flow algorithm, there is an extra $n^{o(1)}$ term in the time complexity.

${ }^{6}$ Note that we actually have to handle a bit more general case where not all distances from $a \in A$ are known to nodes in $B$.
} 
use a very natural framework for APSP. Additionally, if we only care about hop-distances at most $h$, then we can reduce the number of rounds to $\tilde{O}(|A|+h)$. We hope that just as Bellman-Ford is often used as a primitive that allows one to separately handle shorter and longer hop-distances, our new algorithm for DistThrough can be used as a primitive in other distributed shortest path algorithms.

Remark. Throughout the paper we only show that the output is correct with high probability. As discussed in [HNS17], this can be made Las Vegas since in $\tilde{O}(n)$ time we can check the correctness, as follows. First, every node lets its neighbors know about its distances from other nodes (this takes $O(n)$ time). Then, every node checks if it can improve its distance from any node using the distance knowledge from neighbors. If the answer is "no" for every node, then the computed distance is correct. If some node answers "yes", it can broadcast its answer to all other nodes in $O(n)$ time.

\section{High-Level Overview}

We start with a randomized hierarchy: for every integer $1 \leq i \leq \log (n)$, we construct set $S_{i}$ by independently sampling each vertex with probability $1 / 2^{i}$; we set $S_{0}=V$ and $S_{\log (n)+1}=\emptyset$. Then with high probability: $\left|S_{i}\right|=\tilde{O}\left(n / 2^{i}\right)$, and any shortest path with at least $\tilde{O}\left(2^{i}\right)$ vertices contains a vertex from $S_{i}$.

Our algorithm then proceeds in phases, following a standard framework for shortest path algorithms. We go from phase $i=\log (n)$ down to phase 0 . The guarantee at the end of phase $i+1$ is that every vertex $v$ knows the shortest distances from each $s \in S_{i+1}$; that is, $d_{i+1}^{v}=\operatorname{dist}(s, v)$. Let us now consider phase $i$. The goal is for every node $v$ to learn all distances $\operatorname{dist}(s, v)$ for $s \in S_{i}$ and $v \in V$. First, each vertex $s$ in $S_{i}$ runs Bellman-Ford up to hop-distance $\tilde{O}\left(2^{i}\right)$ : this gives us all distances $\operatorname{dist}(s, v)$ for which $h o p(s, v)=\tilde{O}\left(2^{i}\right)$. On the other hand, if $\operatorname{hop}(s, v)$ is large, then we know that there exists a vertex $s_{i+1} \in S_{i+1}$ on the shortest path $\pi(s, v)$. Note, moreover, that because of phase $i+1$ we already know $\operatorname{dist}\left(s_{i+1}, v\right)$; it is also not hard to ensure that we know $\operatorname{dist}\left(s, s_{i+1}\right)$ because of the Bellman-Ford computation from $s_{i}$.

Thus, to complete the phase $i$, all we have left is to solve the sub-problem $\operatorname{Dist}^{-} \operatorname{Through}_{S_{i+1}}\left(S_{i}, V\right)$ : we assume that we already know distances from $S_{i}$ to $S_{i+1}$ and from $S_{i+1}$ to $V$, and the goal is to compute DistThrough $S_{i+1}(s, v)=\min _{s_{i+1} \in S_{i+1}} \operatorname{dist}\left(s, s_{i+1}\right)+\operatorname{dist}\left(s_{i+1}, v\right)$ for every $s \in S_{i}$ and $v \in V$. (In fact the Bellman-Ford computation from each $s \in S_{i}$ only gives us accurate distances to some of the $S_{i+1}$, but this ends up having no effect, so for this overview we stick to the simpler description above.)

Note that DistThrough is a very natural problem in and of itself, and also comes up in many other shortest path algorithms. The issue is that it is not clear how to approach this problem in the distributed setting. The naive solution would be to have each $s_{i} \in S_{i+1}$ broadcast dist $\left(s, s_{i+1}\right)$ for each $s \in S_{i+1}$. But this incurs a congestion of $O\left(\left|S_{i}\right|^{2}\right)$, which is only efficient when $S_{i}$ is relatively small. For this reason, previous algorithms had to deviate from the simple framework described above, and typically tried to balance two different approaches, one for small-hop distances, and one for large ones; in the former case, a Bellman-Ford-style approach is efficient, while for the latter case the relevant $S_{i}$ is small, and so a broadcasting-type-approach is efficient. However, such a trade-off necessarily results in a super-linear round complexity, such as the state of the art of $O\left(n^{1.25}\right)$. 
Our main contribution is to show that $\operatorname{DistThrough}_{B}(A, C)$ can be solved in $\tilde{O}(n)$ time, for any sets $A, B, C \subseteq V$, regardless of their size. Not only does this lead to an optimal round complexity (up to log factors), but it also leads to a very clean and simple solution to the problem, as we are able to use the framework described above, without needing to balance multiple different approaches.

Random Filtered Broadcasting: We solve Dist Through ${ }_{B}(A, C)$ by using a new technique that we refer to as random filtered broadcasting. We focus on a fixed $a \in A$, and show how to solve DistThrough ${ }_{B}(a, C)$ with only $\tilde{O}(1)$ congestion on each edge; using theorem 3.3, we can then parallelize the algorithms for all $a \in A$ in time $\tilde{O}(|A|)=\tilde{O}(n)$. Let us consider the naive broadcasting approach again: each vertex in $b \in B$ knows all distances from $A$, so it sends a message $M(a, b)=(a, \operatorname{dist}(a, b))$ for every $a \in A$. Whenever a vertex $c \in C$ receives message $M(a, b)$, it can use its knowledge of $\operatorname{dist}(b, c)$ to compute $\operatorname{DistThrough}_{b}(a, c)$. Thus, if a vertex $c$ receives $M(a, b)$ for all $b \in B$ it can compute $\operatorname{DistThrough}_{B}(a, c)$.

To reduce the congestion on each edge, we allow vertices to filter out certain message $M(a, b)$, i.e. to not pass them on to their neighbors. Consider the following filtering heuristic: if a vertex $v$ sees a message $M\left(a, b^{\prime}\right)$, but $v$ has previously seen a message $M(a, b)$ with $\operatorname{DistThrough}_{b}(a, v) \leq \operatorname{DistThrough}_{b^{\prime}}(a, v)$, then $v$ does not pass on the message $M\left(a, b^{\prime}\right)$. This reduces the total number of messages sent, and each $c \in C$ still correctly computes Dist Through $_{B}(a, c)$; the reason is that if $b$ is the vertex in $B$ that minimizes $\operatorname{dist}(a, b)+\operatorname{dist}(b, c)$, then it is not hard to see that every node on $\pi(b, c)$ will pass on message $M(a, b)$ (or some equivalently good message, in case of a tie.)

Unfortunately in the worst-case the congestion might be no better than before, as each vertex $v$ might receive the messages $M(a, b)$ in the worst possible order - that is, in decreasing order of $\operatorname{dist}(a, b)+\operatorname{dist}(b, v)$; in this case, $v$ will pass on every message it sees. To overcome this, we use a randomized filter. We let $B_{0}=B$, and obtain each $B_{j}$ by sampling each node in $B_{j-1}$ with probability $1 / 2$. Our algorithm then proceeds in iterations, starting from $j=\log (n)$ down to $j=0$. In iteration $j$, we broadcast all message $M(a, b)$ for $b \in B_{j}$; however, as in the above paragraph, a vertex $v$ filters out messages unless they are strictly better than all previous messages $M\left(a, b^{\prime}\right)$ seen by $v$-i.e. unless $\operatorname{Dist} \operatorname{Through}_{b}(a, v)$ is smaller. The basic argument is that with high probability, $v$ will filter out all but $O(\log (n))$ messages in iteration $j$; the reason is that if we look at the $O(\log (n)) b \in B_{j}$ that are "best" for $v$, then with high probability at least one of them is in $B_{j+1}$, and so was already seen iteration in $j+1$, and will filter out all messages not in the top $O(\log (n))$. We thus have a total congestion of $O(\log (n))$ per iteration, and so $O\left(\log ^{2}(n)\right)$ congestion to compute DistThrough $_{B}(a, C)$, and $\tilde{O}(n)$ time for $\operatorname{DistThrough}_{B}(A, C)$.

\section{Preliminaries}

\subsection{The CONGEST Model}

The communication network is modeled by an undirected unweighted $n$-node $m$-edge graph $G$, where nodes model the processors and edges model the bounded-bandwidth links between the processors. Let $V(G)$ and $E(G)$ denote the set of nodes and (directed) edges of $G$, respectively. The processors (henceforth, nodes) are assumed to have unique IDs in the range of $\{0,1, \ldots, n-1\}$ and infinite computational power. Typically nodes' IDs are assumed to 
be in the range of $\{1, \ldots$, poly $(n)\}$. But as observed in [HNS17], in $O(n)$ time the range can be reduced to $\{0,1, \ldots, n-1\}$. Each node has limited topological knowledge; in particular, it only knows the IDs of its neighbors and knows no other topological information (e.g., whether its neighbors are linked by an edge or not).

Nodes may also accept some additional inputs as specified by the problem at hand. For the case of graph problems, the additional input is typically edge weights. Let $w: E(G) \rightarrow$ $\{1,2, \ldots, \operatorname{poly}(n)\}$ be the edge weight assignment. ${ }^{7}$ We refer to network $G$ with weight assignment $w$ as the weighted network, denoted by $G(w)$. The weight $w(u, v)$ of each edge $(u, v)$ is known only to $u$ and $v$.

We measure the performance of algorithms by its running time, defined as the worst-case number of rounds of distributed communication. At the beginning of each round, all nodes wake up simultaneously. Each node $u$ then sends an arbitrary message of $O(\log n)$ bits through each edge $(u, v)$, and the message will arrive at node $v$ at the end of the round. We assume that nodes always know the number of the current round for simplicity. In this paper, the running time is analyzed in terms of the number of nodes $(n)$. Since $n$ can be computed in $O(D)$ time, where $D$ is the diameter of $G$, we will assume that every node knows $n$.

Remark on edge weights and directions: Note that our algorithm in fact works in the most restricted model studied in the literature, where edge weights are "arbitrary", edges are directed, and communications are unidirectional.

It was commonly assumed in the literature (e.g., [KP08, LPSR09, KP98, GKP98, GK13, HNS17, GL18, FN18]) that the maximum weight is $\operatorname{poly}(n)$; so, each edge weight can be sent through an edge (link) in one round. A more general "arbitrary weight" model has been considered in, e.g., [Elk17b, ARK+18, AR19, AR18]. In this model, edge weights can be arbitrary, and it is assumed that communication links have enough capacity to deliver a distance information in one round. Some algorithms do not work in this model, including the previously best $\tilde{O}\left(n^{5 / 4}\right)$-time algorithm [HNS17].

The case of directed graph has also been studied in the literature. One can consider further whether the communication is bidirectional, i.e. nodes can communicate on an edge regardless of its direction, or the more restricted unidirectional case, where the communication has to be done along edge directions. The previously best $\tilde{O}\left(n^{5 / 4}\right)$-time algorithm [HNS17] has to assume bidirectional communication.

\subsection{Notation and Problem Definition}

Let $G=(V, E)$ be a directed network with arbitrary non-negative weights: $V$ is the set of nodes, and $E$ the set of edges. Let $n=|V|$ and $m=|E|$. Let $(u, v)$ denote the edge from $u$ to $v$, and let $w(u, v)$ be the weight of this edge. For every pair of nodes $s$ and $t$ in $G$, let $\operatorname{dist}(s, t)$ be the shortest distance from $s$ to $t$ in $G$. Note that since the underlying graph $G$ is directed, we might have $\operatorname{dist}(s, t) \neq \operatorname{dist}(t, s)$. Let $\pi(s, t)$ refer to the shortest path from $s$ to $t$; if there are multiple such paths, choose one of the shortest paths with the minimal number of edges. Let hop $(s, t)$ be the number of edges on $\pi(s, t)$.

Throughout the algorithm, each vertex $v$ will maintain for every $u$ various distance estimates $d^{v}(u, v)$. When we refer to such estimates, the superscript $v$ will always refer to

\footnotetext{
${ }^{7}$ Note that it might be natural to include $\infty$ as a possible edge weight. But this is not necessary since it can be replaced by a large weight of value $\operatorname{poly}(n)$.
} 
the node that possesses this knowledge.

Definition 3.1 (All-pairs shortest paths (APSP)). An algorithm for distributed APSP must terminate with every vertex $v \in V$ knowing a value $d^{v}(u, v)=\operatorname{dist}(u, v)$, for every $u \in V$.

We now define a notion of accuracy for the local information at $v$.

Definition 3.2 ( $h$-hop-accurate). For any positive integer $h$, We say that a distance estimate $d^{v}(u, v)$ is h-hop-accurate if the following holds: 1) $d^{v}(u, v) \geq \operatorname{dist}(u, v)$ and 2) if $\operatorname{hop}(x, y) \leq$ $h$ then $d^{v}(u, v)=\operatorname{dist}(u, v)$. Note: if $h \geq n$, then $h$-hop accuracy guarantees $d^{v}(u, v)=$ $\operatorname{dist}(u, v)$.

We say that an event holds with high probability (w.h.p.) if it holds with probability at least $1-1 / n^{c}$, where $c$ is an arbitrarily large constant.

\subsection{Distributed Algorithmic Primitives}

The Bellman-Ford Algorithm. This well-known algorithm computes SSSP from a source $s$ on a network $G$. The algorithm runs for $h$ rounds, where $h$ is an input given by the user. The algorithm offers the following guarantee: upon termination, $d^{t}(s, t)$ is $h$-hop-accurate for every node $t$ in $V$. See Appendix A for a brief description of the algorithm.

Scheduling of Distributed Algorithms. Consider $k$ distributed algorithms $A_{1}, A_{2} \ldots, A_{k}$. Let dilation be such that each algorithm $A_{i}$ finishes in dilation rounds if it runs individually. Let congestion be such that there are at most congestion messages, each of size $O(\log n)$, sent through each edge (counted over all rounds), when we run all algorithms together. We note the following result of Ghaffari [Gha15]:

Theorem 3.3 ([Gha15]). There is a distributed algorithm that can execute $A_{1}, A_{2} \ldots, A_{k}$ altogether in $O($ dilation + congestion $\cdot \log n)$ time.

Negative edge weights: If the original graph has negative weights (and no negative-weight cycles), then we can use the idea of reduced weights from Johnson's algorithm [Joh77] to transform the graph into a new graph with non-negative edge weights that has the same shortest paths as the original graph. The transformation requires $O(n)$ rounds. We can thus assume for the rest of the paper that weights are non-negative. See Appendix B for more details.

\section{The Algorithm}

Define $S_{0}=V$. Let $k=\log (n)$, and for each $i=0, \ldots, k$, select each node to $S_{i}$ with probability $(1 / 2)^{i}$ (every node knows whether it is in $S_{i}$ or not). Let $S_{k+1}=\emptyset$. (Note that we do not require $S_{i+1} \subseteq S_{i}$.) The following facts follow from standard techniques.

Lemma 4.1. W.h.p., the following holds for every $i$.

- $\left|S_{i}\right|=O\left(n \log n / 2^{i}\right)$, and

- for a large enough constant $c$ and for every pairs of nodes $u$ and $v$ such that $h o p(u, v) \geq$ $c 2^{i} \log n$, the shortest path $\pi(u, v)$ contains a node in $S_{i}$. 


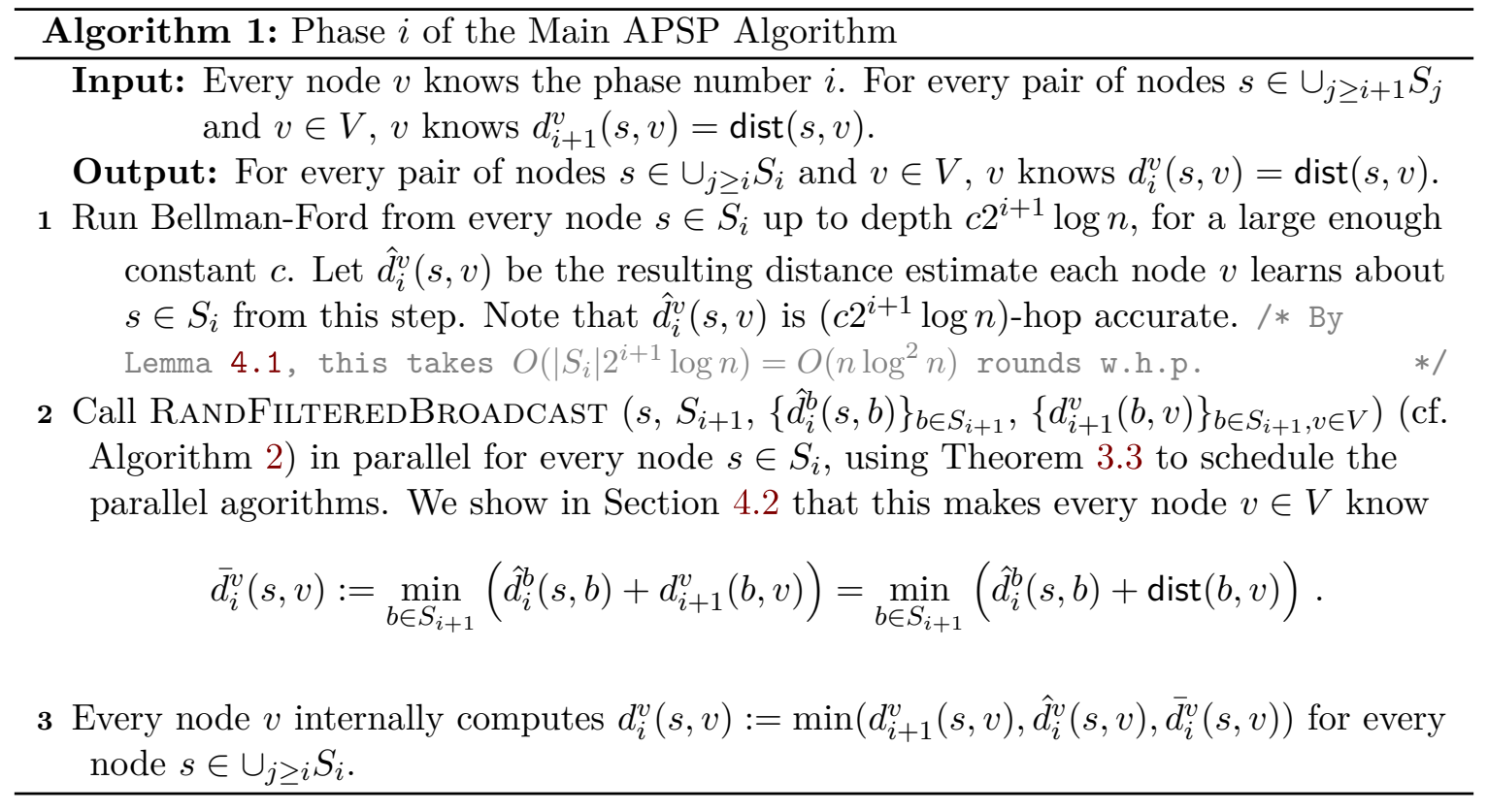

Our algorithm runs in phases, starting from $i=k$ down to 0 . In each phase, we execute Algorithm 1. At the end of phase $i$, every $v \in V$ knows distance $d_{i}^{v}(s, v)=\operatorname{dist}(s, v)$ for every $s \in S_{i}$. Since $S_{0}=V$, the algorithm terminates with knowledge of APSP.

In Algorithm 2, we describe the RANDFilteredBROAdCAST algorithm. Note that in our main algorithm, the set of between-nodes $B$ is always equal to some $S_{i}$; but the RANDFILTEREDBROADCAST subroutine in fact works for an arbitrary set $B$, so we describe in its full generality.

\subsection{Correctness of the Main Algorithm (Algorithm 1)}

In this subsection we show that the output of phase $i$ : for every pair of nodes $s \in \cup_{j \geq i} S_{i}$ and $v \in V, v$ knows $d_{i}^{v}(s, v)=\operatorname{dist}(s, v)$. Since $d_{i+1}^{v}(s, v)=\operatorname{dist}(s, v)$ for every $s \in \cup_{j \geq i+1} S_{j}$, it is enough to show that $d_{i}^{v}(s, v)=\operatorname{dist}(s, v)$ for every $s \in S_{i}$. It is clear that $d_{i}^{v}(s, v) \geq \operatorname{dist}(s, v)$ because every distance returned by our algorithm corresponds to some path in the graph; we now complete the proof by showing that $d_{i}^{v}(s, v) \leq \operatorname{dist}(s, v)$.

Consider any fixed pair $s \in S_{i}$ and $v \in V$, and let $c$ be the constant in Lemma 4.1.

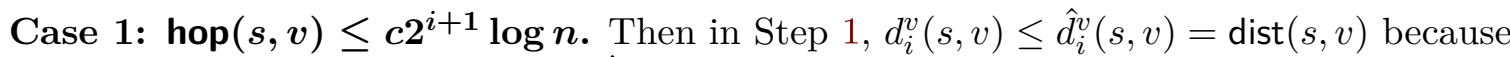
by the properties of Bellman-Ford, $\hat{d}_{i}^{v}(s, v)$ is $c 2^{i+1} \log n$-hop-accurate (see definition 3.2).

Case 2: $\boldsymbol{h o p}(s, \boldsymbol{v})>\boldsymbol{c} \mathbf{2}^{\boldsymbol{i + 1}} \log \boldsymbol{n}$. By Lemma 4.1, there exists a node $b \in S_{i+1}$ that is contained in the shortest path $\pi(s, v)$ and hop $(s, b) \leq c 2^{i+1} \log n$. Bellman-Ford in Step 1 ensures $\hat{d}_{i}^{b}(s, b)=\operatorname{dist}(s, b)$, and since $b \in S_{i+1}$, we have $d_{i+1}^{v}(b, v)=\operatorname{dist}(b, v)$ (by the input assumption for phase $i)$. Thus: $d_{i}^{v}(s, v) \leq \bar{d}_{i}^{v}(s, v) \leq \hat{d}_{i}^{b}(s, b)+d_{i+1}^{v}(b, v)=\operatorname{dist}(s, v)$. 


\subsection{Correctness of RANDFilteredBroadcast (Algorithm 2)}

Lemma 4.2. For any $j \leq \log (n)+1$ and every node $v$, when Iteration $j$ terminates $v$ knows output $^{v}=\min _{b \in B_{j}} \hat{d}^{b}(s, b)+d^{v}(b, v)$; here we define $\min _{b \in \emptyset}(\cdot)=\infty$.

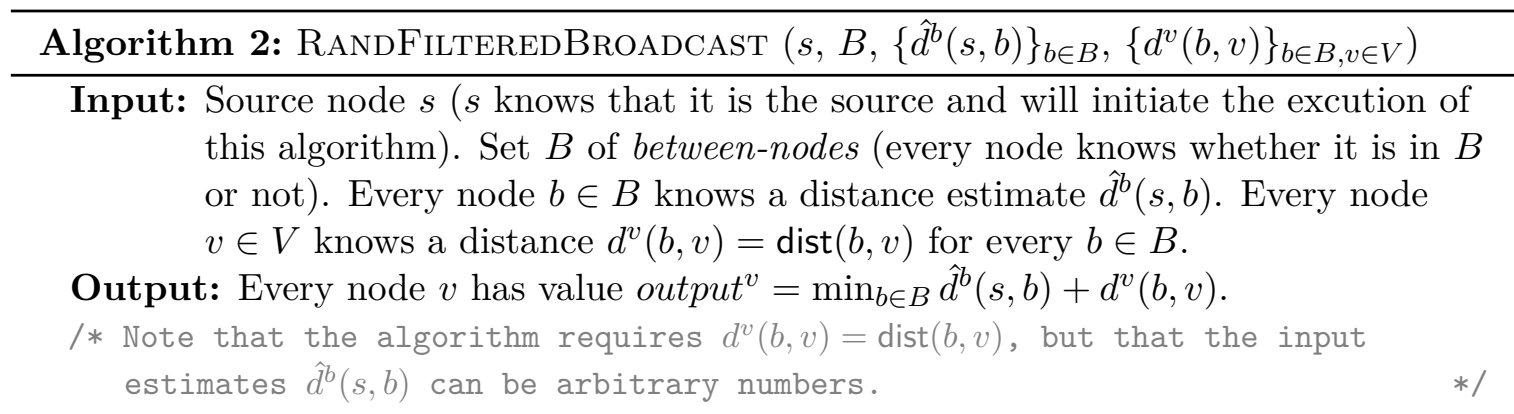

1 Let $B_{0}=B$. For any $j=1, \ldots, \log n$, define $B_{j}$ to be a set where we select each node in $B_{j-1}$ to $B_{j}$ with probability $1 / 2$. Let $B_{\log n+1}=\emptyset$. Note that every node $b \in B$ can decide (randomly) whether or not it is in each $B_{j}$ without any communication.

2 Every node $v$ creates a variable output ${ }^{v}=\infty$.

3 Execute the following in iterations. Starting from Iteration $j=\log (n)$ down to

Iteration $j=0$. Each iteration lasts $n$ rounds. In Iteration $j$, do the following.

(i) Round 1: In parallel, every node $b \in B_{j}$ sets output ${ }^{b}=\hat{d}^{b}(s, b)$ and sends message $M(s, b)=\left(s, b, \hat{d}^{b}(s, b)\right)$ to all neighbors.

/* The $\mathrm{M}(\mathrm{s}, \mathrm{b})$ of round 1 constitute the entire message set of iteraion $j$; future rounds then determine how these messages are passed on.

(ii) At Rounds 2 to $n$, every node $v$ does the following.

(a) Let $B^{v}$ be the set of nodes $b$ such that $v$ has received the message $M(s, b)$. Let $b^{*}$ be the node in $B^{v}$ that minimizes $\hat{d}^{b}(s, b)+d^{v}(b, v)$; ties can be broken arbitrarily.

(b) If $\hat{d}^{b^{*}}\left(s, b^{*}\right)+d^{v}\left(b^{*}, v\right)<$ output $^{v}$, then $v$ sends message $M\left(s, b^{*}\right)$ to all neighbors and sets output ${ }^{v}=\hat{d}^{b^{*}}\left(s, b^{*}\right)+d^{v}\left(b^{*}, v\right)$.

Proof. It is clear that output $t^{v} \geq \min _{b \in B_{j}} \hat{d}^{b}(s, b)+d^{v}(b, v)$, because $v$ only considers values of the form $\hat{d}^{b}(s, b)+d^{v}(b, v)$. The harder direction is to show that output $v^{v} \leq \min _{b \in B_{j}} \hat{d}^{b}(s, b)+$ $d^{v}(b, v)$.

We prove this by induction on $j$. The base case for $j=\log (n)+1$ is trivial, since we define $\min _{b \in \emptyset}(\cdot)=\infty$. For the induction step, we assume that the Lemma holds for $j+1 \leq \log n+1$, and will show that it holds for iteration $j$ as well.

Let us fix some particular node $v$. We now consider two cases; the first is much simpler.

Case 1: $\arg \min _{b \in B_{j}} \hat{\boldsymbol{d}}^{b}(s, \boldsymbol{b})+\boldsymbol{d}^{v}(\boldsymbol{b}, \boldsymbol{v}) \cap \boldsymbol{B}_{j+1} \neq \emptyset$. Intuitively, this is the case that the "best" between-node for $v$ in $B_{j}$ is no better than the best node from $B_{j+1}$; since we know that Lemma 4.2 holds for iteration $j+1$ (inductive hypothesis), the assumption of Case 1 directly ensures that it also holds for iteration $j$.

Case 2: $\arg \min _{b \in B_{j}} \hat{d}^{b}(s, b)+\boldsymbol{d}^{v}(b, v) \cap \boldsymbol{B}_{j+1}=\emptyset$. The rest of the proof is concerned with this case. Let $u_{0}$ be any vertex in $\arg \min _{b \in B_{j}} \hat{d}^{b}(s, b)+d^{v}(b, v)$, and say that $\pi\left(u_{0}, v\right)=$ 
$\left(u_{0}, u_{1}, \ldots u_{\ell}=v\right)$, for some length $\ell$.

Because of the case assumption, we know that for any $b \in B_{j+1}$ we have $\hat{d}^{u_{0}}\left(s, u_{0}\right)+$ $d^{v}\left(u_{0}, v\right)<\hat{d}^{b}(s, b)+d^{v}(b, v)$. Moreover, it is not hard to see that because $\pi\left(u_{0}, v\right)$ is a shortest path, we must also have

$$
\hat{d}^{u_{0}}\left(s, u_{0}\right)+d^{u_{i}}\left(u_{0}, u_{i}\right)<\hat{d}^{b}(s, b)+d^{u_{i}}\left(b, u_{i}\right) \quad \text { for every } b \in B_{j+1} \text { and } u_{i} \in \pi(s, v) .
$$

Now, the intuition behind the proof of Lemma 4.2 is that $v$ should receive the message $M\left(s, u_{0}\right)$, which by choice of $u_{0}$ will ensure that $v$ sets output ${ }^{v} \leq \min _{b \in B_{j}} \hat{d}^{b}(s, b)+d^{v}(b, v)$. The reason we expect this message to travel all the way to $v$ is because by Equation 1, for every $u_{i} \in \pi\left(u_{0}, v\right), u_{0} \in B_{j}$ is a better between node for $u_{i}$ than all $b \in B_{j+1}$, so message $M\left(s, u_{0}\right)$ will pass the filter in Step (ii)b of Algorithm 2. The one issue with this proof is that some $u_{i}$ may fail to pass along $M\left(s, u_{0}\right)$ if it passed along an equally good message $M(s, b)$ in an earlier round of iteration $j$; but this is still fine, as we will show that because $\pi\left(u_{0}, v\right)$ is a shortest path, this message $M(s, b)$ is also good for $v$.

Claim 4.3. For every $r$, by the end of Round $r$ of Iteration $j, u_{r}$ has received a message $M(s, b)$ for some $b$ such that

$$
\hat{d}^{b}(s, b)+d^{u_{r}}\left(b, u_{r}\right) \leq \hat{d}^{u_{0}}\left(s, u_{0}\right)+d^{u_{r}}\left(u_{0}, u_{r}\right) .
$$

Proof. The proof is by induction on the number of $r$. Node $u_{0} \in B_{j}$ sends $M\left(s, u_{0}\right)$ in Round 1 of Iteration $j$, so the claim is obviously true for $u_{1}$. We now assume assume that the claim is true for some $u_{r}$, with $r \geq 1$, and show that the claim must hold for $u_{r+1}$ as well. Let us consider the first message $M(s, b)$ received by $u_{r}$ for which Equation (2) is satisfied; by the induction hypothesis, $u_{r}$ receives this message at some time $t \leq r$, and moreover, by Equation 1, this event first occurs in iteration $j$; it could not have occurred in an earlier iteration $j^{\prime}>j$. Thus, by Step (ii)b of Algorithm 2), we know that at time $t, u_{r}$ set output $t^{u_{r}}$ to $\hat{d}^{b}(s, b)+d^{u_{r}}\left(b, u_{r}\right)$, and sent message $M(s, b)$ to all its neighbors, including $u_{r+1}$

Thus, $u_{r+1}$ receives message $M(s, b)$ at time $t+1 \leq r+1$. Observe that:

$$
\begin{aligned}
& \hat{d}^{b}(s, b)+d^{u_{r+1}}\left(b, u_{r+1}\right) \\
& =\hat{d}^{b}(s, b)+\operatorname{dist}\left(b, u_{r+1}\right) \\
& \leq \hat{d}^{b}(s, b)+\operatorname{dist}\left(b, u_{r}\right)+\operatorname{dist}\left(u_{r}, u_{r+1}\right) \\
& =\hat{d}^{b}(s, b)+d^{u_{r}}\left(b, u_{r}\right)+\operatorname{dist}\left(u_{r}, u_{r+1}\right) \\
& \leq \hat{d}^{u_{0}}\left(s, u_{0}\right)+d^{u_{r}}\left(u_{0}, u_{r}\right)+\operatorname{dist}\left(u_{r}, u_{r+1}\right) \\
& =\hat{d}^{u_{0}}\left(s, u_{0}\right)+\operatorname{dist}\left(u_{0}, u_{r}\right)+\operatorname{dist}\left(u_{r}, u_{r+1}\right) \\
& =\hat{d}^{u_{0}}\left(s, u_{0}\right)+\operatorname{dist}\left(u_{0}, u_{r+1}\right) \\
& \leq \hat{d}^{u_{0}}\left(s, u_{0}\right)+d^{u_{r+1}}\left(u_{0}, u_{r+1}\right)
\end{aligned}
$$

(by input condition of Algorithm 2)

(by triangle inequality)

(by input condition of Algorithm 2)

(by Equation (2))

(by input condition of Algorithm 2)

(since $u_{r}$ is on the shortest $\left(u_{0} u_{r+1}\right)$-path)

(by input condition of Algorithm 2).

Message $M(s, b)$ thus satisfies Equation (2) for node $u_{r+1}$, which completes the induction proof of Claim 4.3

Since each iteration has $n$ rounds, Claim 4.3 implies that node $v$ will receive $M(s, b)$ satisfying Equation (2) by the end of Iteration $j$; thus by the choice of $u_{0}$, Step (ii) b of 
Algorithm 2) sets output ${ }^{v}$ to be at most

$$
\hat{d}^{b}(s, b)+d^{u_{r}}\left(b, u_{r}\right) \leq \hat{d}^{u_{0}}\left(s, u_{0}\right)+d^{u_{r}}\left(u_{0}, u_{r}\right)=\min _{b \in B_{j}} \hat{d}^{b}(s, b)+d^{v}(b, v,)
$$

as desired. This concludes the proof of Lemma 4.2.

Observation 4.4. We do not need this for our main result, but we note that if the input had the additional guarantee that all hop-distances between $B$ and $V$ were at most $h$, then we would only need to run RANDFILTEREDBROADCAST for $h$ rounds instead of $n$; this is because in case 2 of the proof, $h$ rounds would suffice for the message to propagate from $u_{0}$ to $u_{\ell}=v$.

\subsection{Complexity of RANDFilteredBroAdCAST (Algorithm 2)}

The time complexity of Algorithm 2 is clearly $O(n \log (n))$, since there are $O(\log (n))$ iterations, and each is specified to run for $O(n)$ rounds. Now we show that the algorithm creates low congestion on every edge, and thus can be easily parallelized.

Lemma 4.5. W.h.p., every node $v$ sends to its neighbors $O(\log n)$ messages of the form $M(s, b)$ in each iteration $j$ of Algorithm 2.

Proof. The claim is true for $j=\log n$ because by lemma 4.1, $\left|B_{\log n}\right|=O(\log n)$ w.h.p. Now, Consider any Iteration $j<\log n$ of Algorithm 2 and any node $v$. Define for any node $b \in B$

$$
B_{j}^{\prime}(b, v)=\left\{b^{\prime} \in B_{j} \mid \hat{d}^{b^{\prime}}\left(s, b^{\prime}\right)+d^{v}\left(b^{\prime}, v\right) \leq \hat{d}^{b}(s, b)+d^{v}(b, v)\right\} .
$$

Observe that if $B_{j}^{\prime}(b, v) \cap B_{j+1} \neq \emptyset$, then $v$ will not send $M(s, b)$ to neighbors in Step (ii)b of Iteration $j$, because by lemma 4.2 , at the end of the previous iteration $(j+1)$ we will already have output ${ }^{v}=\min _{b^{\prime} \in B_{j+1}}\left(\hat{d}^{b}\left(s, b^{\prime}\right)+d^{v}\left(b^{\prime}, v\right)\right) \leq \hat{d}^{b}(s, b)+d^{v}(b, v)$. Observe further that the definition of $B_{j}^{\prime}(b, v)$ does not depend on the randomness used to sample $B_{j+1}$; thus, since each $b \in B_{j}$ is sampled into $B_{j+1}$ with probability $1 / 2$, we have:

$$
\forall \gamma>0, \forall\left|B_{j}^{\prime}(b, v)\right| \geq \gamma \log n, \operatorname{Pr}\left[B_{j}^{\prime}(b, v) \cap B_{j+1}=\emptyset\right]=1 / 2^{\left|B_{j}^{\prime}(b, v)\right|}=O\left(n^{-\gamma}\right),
$$

Applying a union bound over the $\leq n$ possible values of $b$, we get: w.h.p, for all messages $M(s, b)$ sent by $v$ with $b \in B_{j}$, we have that $\left|B_{j}^{\prime}(b, v)\right|=O(\log n)$. Lemma 4.5 follows from the fact that there are $O(\log n)$ nodes $b \in B_{j}$ with $\left|B_{j}^{\prime}(b, v)\right|=O(\log n)$. To see this, order nodes $b$ in $B_{j}$ by increasing values of $\hat{d}^{b}(s, b)+d^{v}(b, v)$ (break ties arbitrarily). Observe that for the $i^{\text {th }}$ node $b$ in this order, $B_{j}^{\prime}(b, v)$ contains all nodes that appear before $b$ in the order. Thus, only the first $O(\log (n))$ in this order have the property that $\left|B_{j}^{\prime}(b, v)\right|=O(\log n)$.

Corollary 4.6. Over all $\log (n)$ iterations, Algorithm 2 terminates in $O(n \log (n))$ rounds, and incurs a congestion of $O\left(\log ^{2}(n)\right)$ on each edge.

\subsection{Complexity of the Main Algorithm (Algorithm 1)}

Recall that Algorithm 1 runs in $k=\log (n)$ phases. We now analyze the complexity of an individual phase: summing over all the phases completes the proof of our main result. 
Lemma 4.7. W.h.p phase $i$ of Algorithm 1 terminates in $O\left(n \log ^{2}(n)+\left|S_{i}\right| \log ^{3}(n)\right)=$ $O\left(n \log ^{2}(n)+n \log ^{4}(n) / 2^{i}\right)$ rounds

Proof. By Lemma 4.1 and the properties of Bellman-Ford, Step 1 of Algorithm 1 requires a total of $O\left(\left|S_{i}\right| 2^{i+1} \log n\right)=O\left(n \log ^{2} n\right)$ rounds w.h.p.

Step 3 of Algorithm 1 does not require any communication, so all that remains is to analyze the number of rounds required for all the calls to RANDFILTEREDBROADCAST in Step 2. The algorithm runs $\left|S_{i}\right|$ instances of RANDFilteredBroadCAST in parallel. By corollary 4.6 each runs in $O(n \log (n))$ rounds and incurs $O\left(\log ^{2}(n)\right)$ congestion per edge. Thus the total congestion is $O\left(\left|S_{i}\right| \log ^{2}(n)\right)$, so using the parallel scheduler in theorem 3.3 yields a total round complexity of $O\left(\left|S_{i}\right| \log ^{3}(n)\right)$, as desired.

\section{Open Problems}

As mentioned earlier, deterministic $\tilde{O}(n)$-time algorithms for APSP remains a key open problem. Additionally, while APSP admits an $\tilde{\Omega}(n)$ lower bound, it is a curious question whether this bound also holds for the following strongly connected component problem: We want every node to output a "label" such that two nodes are in the same strongly-connected component if and only if their labels are the same (or even simpler, just counting the number of connected components). It should also be interesting to see how our algorithm performs in real systems (such as D-Galois [HPD+19]), and to see if our ideas are useful in computing various centrality measures (e.g. [HPD+19]).

A few problems remain open for SSSP. An obvious one is closing the gap between lower and upper bounds for SSSP [FN18, DHK+12] and the single-source reachability problem [GU15]. Another question is whether the best upper bound for SSSP can be obtained without the scaling technique, so that we can avoid the dependency on the ratio between the highest and lowest edge weights. Recall that the previous state-of-the-art algorithms for both APSP and SSSP [HNS17, GL18, FN18] require this technique, but our algorithm does not.

This paper is part of an effort to understand exact distributed graph algorithms, and more generally to classify complexities of global problems in the CONGEST model. Many problems are yet to be settled, including minimum cut [DHN+19], maximum weight/cardinality matching [AKO18], st-cut/flow [GKK+15], vertex connectivity [CGK14], and densest subgraph $[\mathrm{DLN}+12]$. As mentioned earlier, settling the exact cases for other problems remains a major open problem. As mentioned in [CKP17, DHN+19], tight bounds witnessed so far are in the form of either $\tilde{\Theta}(D), \tilde{\Theta}(\sqrt{n}+D), \tilde{\Theta}(n)$, or $\tilde{\Theta}\left(n^{2}\right)$. Any tight bound in-between is of our interest.

Finally, we propose studying the relationship between the node-partition two-party communication complexity and distributed graph algorithms in the CONGEST model. The only known technique to prove a lower bound of $\tilde{\Omega}(t)$ for any $t \geq n$ (e.g. [FHW12, ACK16, CKP17, Nan14]) in the CONGEST model is to partition nodes into two sides and argue (via two-party communication complexity-theoretic arguments) that there must be $\tilde{\Omega}(t|C|$ ) bits of information between the two sides, where $C$ is the set of edges between the two sides. (See, e.g., [CKP17] for details.) Is this the only technique for proving superlinear lower bounds? In particular, experiences from APSP (where this technique was shown incapable of proving a superlinear lower bound [CKP17] before we settle a near-linear upper bound here) make it tempting to conjecture that if there is a protocol $\mathcal{A}$ with $\tilde{O}(t|C|)$ total 
communication for solving any graph problem $P$ in the two-party model above, then there is an $\tilde{O}(t)$-time CONGEST algorithm $\mathcal{B}$ for $P$, for any $t \geq n$. This conjecture sounds too good to be true in general. It will be extremely exciting already if it holds for some natural class of graph problems, even just for some $t \in\left(\sqrt{n}, n^{2}\right)$. A related, more plausible, conjecture is to consider when $\mathcal{A}$ takes only $\tilde{O}(t)$ rounds.

\section{Acknowledgement}

This project has received funding from the European Research Council (ERC) under the European Union's Horizon 2020 research and innovation programme under grant agreement No 715672. Nanongkai was also partially supported by the Swedish Research Council (Reg. No. 2015-04659.)

\section{References}

[ACK16] Amir Abboud, Keren Censor-Hillel, and Seri Khoury. "Near-Linear Lower Bounds for Distributed Distance Computations, Even in Sparse Networks". In: DISC. Vol. 9888. Lecture Notes in Computer Science. Springer, 2016, pp. 29-42 (cit. on pp. 1, 11).

[AKO18] Mohamad Ahmadi, Fabian Kuhn, and Rotem Oshman. "Distributed Approximate Maximum Matching in the CONGEST Model". In: DISC. Vol. 121. LIPIcs. Schloss Dagstuhl - Leibniz-Zentrum fuer Informatik, 2018, 6:1-6:17 (cit. on p. 11).

[AR18] Udit Agarwal and Vijaya Ramachandran. "New and Simplified Distributed Algorithms for Weighted All Pairs Shortest Paths". In: CoRR abs/1810.08544 (2018) (cit. on pp. 1, 2, 5).

[AR19] Udit Agarwal and Vijaya Ramachandran. "A Deterministic Distributed Algorithm for Weighted All Pairs Shortest Paths Through Pipelining". In: IPDPS (2019) (cit. on pp. 1, 2, 5).

[ARK+18] Udit Agarwal, Vijaya Ramachandran, Valerie King, and Matteo Pontecorvi. "A Deterministic Distributed Algorithm for Exact Weighted All-Pairs Shortest Paths in Õ(n 3/2 ) Rounds". In: PODC. ACM, 2018, pp. 199-205 (cit. on pp. 1, $2,5)$.

$[\mathrm{BKK}+17]$ Ruben Becker, Andreas Karrenbauer, Sebastian Krinninger, and Christoph Lenzen. "Near-Optimal Approximate Shortest Paths and Transshipment in Distributed and Streaming Models". In: DISC. Vol. 91. LIPIcs. Schloss Dagstuhl - Leibniz-Zentrum fuer Informatik, 2017, 7:1-7:16 (cit. on p. 2).

[Bel58] Richard Bellman. "On a Routing Problem". In: Quarterly of Applied Mathematics 16.1 (1958), pp. 87-90 (cit. on p. 15).

[CGK14] Keren Censor-Hillel, Mohsen Ghaffari, and Fabian Kuhn. "Distributed connectivity decomposition". In: PODC. ACM, 2014, pp. 156-165 (cit. on p. 11).

[CKP17] Keren Censor-Hillel, Seri Khoury, and Ami Paz. "Quadratic and Near-Quadratic Lower Bounds for the CONGEST Model". In: DISC. 2017 (cit. on pp. 1, 11). 
[DHK+12] Atish Das Sarma, Stephan Holzer, Liah Kor, Amos Korman, Danupon Nanongkai, Gopal Pandurangan, David Peleg, and Roger Wattenhofer. "Distributed Verification and Hardness of Distributed Approximation". In: SIAM Journal on Computing 41.5 (2012). Announced at STOC'11, pp. 1235-1265 (cit. on pp. 2, 11).

[DHN+19] Mohit Daga, Monika Henzinger, Danupon Nanongkai, and Thatchaphol Saranurak. "Distributed Edge Connectivity in Sublinear Time". In: STOC. ACM, 2019 (cit. on p. 11).

$[\mathrm{DLN}+12]$ Atish Das Sarma, Ashwin Lall, Danupon Nanongkai, and Amitabh Trehan. "Dense Subgraphs on Dynamic Networks". In: DISC. Vol. 7611. Lecture Notes in Computer Science. Springer, 2012, pp. 151-165 (cit. on p. 11).

$[$ EKN+14] Michael Elkin, Hartmut Klauck, Danupon Nanongkai, and Gopal Pandurangan. "Can Quantum Communication Speed Up Distributed Computation?" In: Symposium on Principles of Distributed Computing (PODC). 2014, pp. 166-175 (cit. on p. 2).

[Elk06] Michael Elkin. "An Unconditional Lower Bound on the Time-Approximation Trade-off for the Distributed Minimum Spanning Tree Problem". In: SIAM Journal on Computing 36.2 (2006). Announced at STOC'04, pp. 433-456 (cit. on p. 2).

[Elk17a] Michael Elkin. "A Simple Deterministic Distributed MST Algorithm, with Near-Optimal Time and Message Complexities". In: CoRR abs/1703.02411 (2017) (cit. on p. 2).

[Elk17b] Michael Elkin. "Distributed Exact Shortest Paths in Sublinear Time". In: Symposium on Theory of Computing, STOC. 2017 (cit. on pp. 1, 2, 5).

[FHW12] Silvio Frischknecht, Stephan Holzer, and Roger Wattenhofer. "Networks cannot compute their diameter in sublinear time". In: SODA. 2012, pp. 1150-1162 (cit. on pp. 1, 11).

[FN18] Sebastian Forster and Danupon Nanongkai. "A Faster Distributed Single-Source Shortest Paths Algorithm”. In: FOCS. IEEE Computer Society, 2018, pp. 686697 (cit. on pp. 2, 5, 11).

[For56] Lester R. Ford. Network Flow Theory. Tech. rep. P-923. The Rand Corporation, 1956 (cit. on p. 15).

[GK13] Mohsen Ghaffari and Fabian Kuhn. "Distributed Minimum Cut Approximation". In: Symposium on Distributed Computing (DISC). 2013, pp. 1-15 (cit. on pp. 2, $5)$.

[GKK+15] Mohsen Ghaffari, Andreas Karrenbauer, Fabian Kuhn, Christoph Lenzen, and Boaz Patt-Shamir. "Near-Optimal Distributed Maximum Flow: Extended Abstract". In: Proceedings of the 2015 ACM Symposium on Principles of Distributed Computing, PODC 2015, Donostia-San Sebastián, Spain, July 21 - 23, 2015. 2015, pp. 81-90 (cit. on pp. 2, 11). 
[GKP98] Juan A. Garay, Shay Kutten, and David Peleg. "A Sublinear Time Distributed Algorithm for Minimum-Weight Spanning Trees". In: SIAM Journal on Computing 27.1 (1998). Announced at FOCS'93, pp. 302-316 (cit. on p. 5).

[GL18] Mohsen Ghaffari and Jason Li. "Improved distributed algorithms for exact shortest paths". In: STOC. ACM, 2018, pp. 431-444 (cit. on pp. 2, 5, 11).

[GU15] Mohsen Ghaffari and Rajan Udwani. "Brief Announcement: Distributed SingleSource Reachability". In: Proceedings of the 2015 ACM Symposium on Principles of Distributed Computing, PODC 2015, Donostia-San Sebastián, Spain, July 21 - 23, 2015. 2015, pp. 163-165 (cit. on p. 11).

[Gha15] Mohsen Ghaffari. "Near-Optimal Scheduling of Distributed Algorithms". In: Proceedings of the 2015 ACM Symposium on Principles of Distributed Computing, PODC 2015, Donostia-San Sebastián, Spain, July 21 - 23, 2015. 2015, pp. 3-12 (cit. on p. 6).

[HKN16] Monika Henzinger, Sebastian Krinninger, and Danupon Nanongkai. "A deterministic almost-tight distributed algorithm for approximating single-source shortest paths". In: Proceedings of the 48th Annual ACM SIGACT Symposium on Theory of Computing, STOC 2016, Cambridge, MA, USA, June 18-21, 2016. 2016, pp. 489-498 (cit. on p. 2).

[HNS17] Chien-Chung Huang, Danupon Nanongkai, and Thatchaphol Saranurak. "Distributed Exact Weighted All-Pairs Shortest Paths in O $\left(\mathrm{n}^{5 / 4}\right)$ Rounds". In: FOCS. IEEE Computer Society, 2017, pp. 168-179 (cit. on pp. 1-3, 5, 11).

[HPD+19] Loc Hoang, Matteo Pontecorvi, Roshan Dathathri, Gurbinder Gill, Bozhi You, Keshav Pingali, and Vijaya Ramachandran. "A round-efficient distributed betweenness centrality algorithm". In: PPoPP. ACM, 2019, pp. 272-286 (cit. on p. 11).

[HW12] Stephan Holzer and Roger Wattenhofer. "Optimal Distributed All Pairs Shortest Paths and Applications". In: Symposium on Principles of Distributed Computing (PODC). 2012, pp. 355-364 (cit. on pp. i, 1).

[Joh77] Donald B. Johnson. "Efficient Algorithms for Shortest Paths in Sparse Networks". In: J. ACM 24.1 (1977), pp. 1-13 (cit. on pp. 6, 16).

[KKP13] Liah Kor, Amos Korman, and David Peleg. "Tight Bounds for Distributed Minimum-Weight Spanning Tree Verification". In: Theory of Computing Systems 53.2 (2013). Announced at STACS'11, pp. 318-340 (cit. on p. 2).

[KP08] Maleq Khan and Gopal Pandurangan. "A fast distributed approximation algorithm for minimum spanning trees". In: Distributed Computing 20.6 (2008). Announced at DISC'06, pp. 391-402 (cit. on p. 5).

[KP98] Shay Kutten and David Peleg. "Fast Distributed Construction of Small $k$ Dominating Sets and Applications". In: Journal of Algorithms 28.1 (1998). Announced at PODC'95, pp. 40-66 (cit. on pp. 2, 5).

[LP13] Christoph Lenzen and David Peleg. "Efficient Distributed Source Detection with Limited Bandwidth". In: Symposium on Principles of Distributed Computing (PODC). 2013, pp. 375-382 (cit. on pp. i, 1). 
[LP15] Christoph Lenzen and Boaz Patt-Shamir. "Fast Partial Distance Estimation and Applications". In: Symposium on Principles of Distributed Computing (PODC). 2015, pp. 153-162 (cit. on pp. 1, 2).

[LPS13] Christoph Lenzen and Boaz Patt-Shamir. "Fast Routing Table Construction Using Small Messages". In: Symposium on Theory of Computing (STOC). 2013, pp. 381-390 (cit. on pp. 1, 2).

[LPSR09] Zvi Lotker, Boaz Patt-Shamir, and Adi Rosén. "Distributed Approximate Matching". In: SIAM J. Comput. 39.2 (2009), pp. 445-460 (cit. on p. 5).

[NS14] Danupon Nanongkai and Hsin-Hao Su. "Almost-Tight Distributed Minimum Cut Algorithms". In: International Symposium on Distributed Computing (DISC). 2014, pp. 439-453 (cit. on p. 2).

[Nan14] Danupon Nanongkai. "Distributed Approximation Algorithms for Weighted Shortest Paths". In: Symposium on Theory of Computing (STOC). 2014, pp. 565573 (cit. on pp. i, 1, 2, 11).

[PR00] David Peleg and Vitaly Rubinovich. "A Near-Tight Lower Bound on the Time Complexity of Distributed Minimum-Weight Spanning Tree Construction". In: SIAM Journal on Computing 30.5 (2000). Announced at FOCS'99, pp. 14271442 (cit. on p. 2).

[PRS17] Gopal Pandurangan, Peter Robinson, and Michele Scquizzato. "A Time- and Message-Optimal Distributed Algorithm for Minimum Spanning Trees". In: Symposium on Theory of Computing, STOC. 2017 (cit. on p. 2).

[PRT12] David Peleg, Liam Roditty, and Elad Tal. "Distributed Algorithms for Network Diameter and Girth". In: ICALP (2). 2012, pp. 660-672 (cit. on pp. i, 1).

[Pel00] David Peleg. Distributed Computing: A Locality-sensitive Approach. Philadelphia, PA, USA: Society for Industrial and Applied Mathematics, 2000. ISBN: 0-89871-464-8 (cit. on p. 16).

\section{Appendix}

\section{A Bellman-Ford}

Since it figures prominently in our main algorithm, we now describe the well-known BellmanFord algorithm for computing SSSP from a source $s$ on network $G$ [Bel58, For56]. We omit the analysis of the algorithm, since it can be found in the citations. The algorithm runs for $h$ rounds, where $h$ is an input given by the user.

For any node $t$, let $d^{t}(s, t)$ denote the knowledge of $t$ about $\operatorname{dist}(s, t)$. Initially, $d^{t}(s, t)=\infty$ for every node $t$, except that $d^{s}(s, s)=0$. The algorithm proceeds as follows.

(i) In round 0 , every node $t$ sends $d^{t}(s, t)$ to all its neighbors.

(ii) When a node $t$ receives the message about $d^{x}(s, x)$ from its neighbors $x$, it uses the new information to decrease the value of $d^{t}(s, t)$ if $d^{x}(s, x)+w(x, t)<d^{t}(s, t)$.

(iii) If $d^{t}(s, t)$ decreases, then node $t$ sends the new value of $d^{t}(s, t)$ to all its neighbors.

(iv) Repeat (ii) and (iii) for $h$ rounds. 
Clearly, the above algorithm takes $O(h)$ rounds. Moreover, it can be proved that when the algorithm terminates $d^{t}(s, t)$ is $h$-hop-accurate for every node $t$ in $V$.

\section{B Non-negative weights}

In this section we show that if the original graph has negative weights but no non-negative cycles, we can in $O(n)$ rounds transform it to a graph that has exactly the same shortest path structure, but has non-negative weights. This justifies the assumption of non-negative weights in section 3. (If the graph has a negative cycle, then the algorithm will discover this cycle within $O(n)$ rounds.)

Our transformation directly follows the technique of reduced costs used in Johnson's APSP algorithm in the static setting [Joh77]. The algorithm will compute a node value $\phi(v)$ for every node $v$ such that the following property is satisfied: for every edge $(x, y) \in E$, $\phi(x)+w(x, y)-\phi(y) \geq 0$. We show how to compute the values $\phi(v)$ later. Once these values are computed, the algorithm creates a new edge-weight function $w^{\prime}: E \rightarrow \mathbb{R}_{\geq 0}$, where $w^{\prime}(x, y)=\phi(x)+w^{\prime}(x, y)-\phi(y)$. Let $G^{\prime}=\left(V, E^{\prime}\right)$ the graph with the weight function $w^{\prime}$ instead of $w$, and let $\operatorname{dist}^{\prime}(s, t)$ be the shortest $s-t$ distance in $G^{\prime}$. It is to easy to see that $G^{\prime}$ satisfies the following properties:

1. $w^{\prime}(u, v) \geq 0$ for every edge $(u, v)$.

2. for every pair of nodes $s$ and $t$ we have $\operatorname{dist}(s, t)=\operatorname{dist}^{\prime}(s, t)+\phi(t)-\phi(s)$.

Thus overall algorithm proceeds as follows. First it executes process COMPUTE- $\phi$, described below: at the end of this process, each vertex $v$ knows its own value $\phi(v)$. Then each vertex $v$ broadcasts $\phi(v)$ to the entire graph: by lemma B.1, this takes a total of $O(n)$ rounds.

Lemma B.1 (Broadcasting [Pel00]). Suppose each $v \in V$ holds $k_{v} \geq 0$ messages of $O(\log n)$ bits each, for a total of $K=\sum_{v \in V} k_{v}$ messages. Then all nodes in the network can receive these $K$ messages within $O(K+D)$ rounds.

The algorithm then executes the main distributed APSP algorithm described in this paper on on $G^{\prime}$ instead of $G$ : by Property 1 of $G^{\prime}$ it only encounters non-negative weights, as desired. When the APSP algorithm on $G^{\prime}$ terminates, the guarantee is that for every pair of nodes $s$ and $t$, node $t$ knows dist ${ }^{\prime}(s, t)$. By Property 2 of $G^{\prime}, t$ can then figure out $\operatorname{dist}(s, t)$ using its knowledge of $\operatorname{dist}(s, t), \phi(s)$ and $\phi(t)$.

All we have left to show is how to execute COMPUTE- $\phi$. Let the graph $G^{*}$ be the original graph $G$, but with an additional vertex $s^{*}$, and a directed edge of weight 0 from $s^{*}$ to every node $v$. The algorithm then sets $\phi(v)=\operatorname{dist}\left(s^{*}, v\right)$ for every $v$. It is not hard to check that because of the triangle inequality for shortest distances, we have $\phi(x)+w(x, y)-\phi(y) \geq 0$, as desired. We can compute $\operatorname{dist}\left(s^{*}, v\right)$ for every vertex $v$ by simply running Bellman-Ford for $n$ rounds: to deal with the fact that vertex $s^{*}$ does not actually exist, the algorithm executes Bellman Ford exactly as described in section 3.3, except that in step i) it initializes $\operatorname{dist}\left(s^{*}, v\right)=0$ for every node $v$. Note that Bellman-Ford will also detect if there exists a negative weight cycle in the graph. 